\title{
Two unusual cases of intra-atrial gap
}

\author{
A. WALEFFE, P. BRUNINX, J-C. DEMOULIN, AND H. E. KULBERTUS \\ From the Division of Cardiology, Institute of Medicine, University of Liège School of Medicine, Liège, \\ Belgium
}

Two cases are described in which, during investigation of rhythm disturbances, it was shown that, by delivering atrial stimuli of increasing prematurity, a point was reached where the artificial stimulus failed to induce an atrial response. However, with even more premature stimulations, atrial responses were again observed. These observations are best explained by a gap phenomenon in which very early stimuli delivered during the effective refractory period of the atria were delayed long enough in the exit area around the pacing electrode to allow atrial recovery.

The phenomenon of 'gap' in atrioventricular conduction was first described by Moe et al. (1965). During experiments on dogs, they observed a zone in the cardiac cycle when atrial premature beats were not transmitted to the ventricles whereas atrial beats of greater or lesser prematurity were normally conducted to the ventricles. The atrioventricular gap phenomenon occurs when the effective refractory period of a distal site (for example, the His-Purkinje system) is longer than the functional refractory period of a proximal site (for example the AV node), and when closely coupled stimuli are delayed long enough at the proximal site to allow recovery at the distal end (Wu et al., 1974).

At present, at least 6 types of gap phenomenon in atrioventricular conduction have been recognised; they differ from one another in the sites of proximal delay and distal block (Damato et al., 1976).

In this paper, we report on two patients in whom the electrophysiological findings strongly suggested an unusual gap phenomenon where the exit area around a pacing electrode was the site of proximal delay and the atria the distal site with relatively long effective refractory period.

\section{Methods}

Electrophysiological investigations were carried out, after informed consent, on 2 patients with rhythm disturbances. The studies were performed in the supine, non-sedated, post-absorptive state. His bundle recordings were obtained by the technique described by Scherlag et al. (1969), Received for publication 5 July 1976 using a bipolar electrode catheter introduced percutaneously into the femoral vein. Two other bipolar electrode catheters were employed, the first to stimulate the high lateral wall of the right atrium and the second to record the high right atrial electrogram.

The His bundle electrogram, high right atrial electrogram, and 2 surface leads were recorded simultaneously on a 4-channel Elema Mingograf 81 at a paper speed of $100 \mathrm{~mm} / \mathrm{s}$. The stimuli were $2 \mathrm{~ms}$ square waves approximately equal to twice diastolic threshold and were delivered using a JS-U2 multipurpose programmable stimulator.

During programmed stimulation, the test stimuli were given after each eighth driving stimulus or spontaneous beat, the coupling interval being decreased by decrements of $5 \mathrm{~ms}$.

\section{Results}

CASE 1

The patient was a 56-year-old man who had had an acute anterolateral myocardial infarct in September 1975. He was admitted in January 1976 for treatment of cardiac failure with digitalis and diuretics. Investigations at that time showed a large parietal left ventricular aneurysm, a totally occluded left anterior descending artery with poor distal perfusion, and significantly stenosed left circumflex and right coronary arteries. He had recurrent episodes of ventricular tachycardia for which an electrophysiological study was undertaken about one month later.

During sinus rhythm at $100 / \mathrm{min}$ the $\mathrm{AH}$ and HV intervals were $130 \mathrm{~ms}$ and $40 \mathrm{~ms}$, respectively. 
The so-called sinoatrial conduction time was $62.5 \mathrm{~ms}$.

Atrial premature stimuli were introduced at a cycle length of $400 \mathrm{~ms}$ (Fig. 1 and 2). As the S1-S2 interval was decreased from 360 to $290 \mathrm{~ms}$, the HRA1-HRA2 decreased similarly. When the S1-S2 interval was further shortened, the HRA1HRA2 intervals were seen to leave the line of identity, thus indicating increased S2-HRA2 delay. At S1-S2 intervals of 260, 255, 250, and $245 \mathrm{~ms}$, S2 was followed by no atrial response though the driving stimulus (S3) falling immediately after S2, did induce an atrial depolarisation. Surprisingly, the S2 stimuli placed 240 and $235 \mathrm{~ms}$ after $S 1$ were followed by atrial contractions, but the S2-HRA2 intervals were prolonged and the two corresponding HRA1-HRA2 intervals were approximately equal to those recorded immediately before the gap.

CASE 2

Case 2 was a 58-year-old woman who had had an asymptomatic bradycardia for several years. In 1975, her electrocardiogram showed a junctional rhythm at $53 / \mathrm{min}$ with ventricular ectopic beats. Isoprenaline, administered orally, produced a slight increase in heart rate, but was poorly tolerated. In March 1976, she complained of dyspnoea and was found to be hypertensive $(220 / 100 \mathrm{mmHg})$. The electrocardiogram still showed sinus bradycardia alternating with junctional rhythm.

The electrophysiological investigation yielded the following results. At rest, at a basic cycle length of $960 \mathrm{~ms}$, the AH and $\mathrm{HV}$ intervals were 90 and $45 \mathrm{~ms}$, respectively. The sinoatrial conduction time could not be measured, no plateau zone being found on the curve plotting the return cycles against the test cycles. Atrial pacing at $120 / \mathrm{min}$ and $140 / \mathrm{min}$ was followed by cardiac pauses longer than $2000 \mathrm{~ms}$. A diagnosis of sinoatrial disease was thus made.

Atrial premature stimuli were introduced at a cycle length of $545 \mathrm{~ms}$ (Fig. 3 and 4). The curve obtained by plotting the S1-S2 intervals against the HRA1-HRA2 intervals followed the line of identity

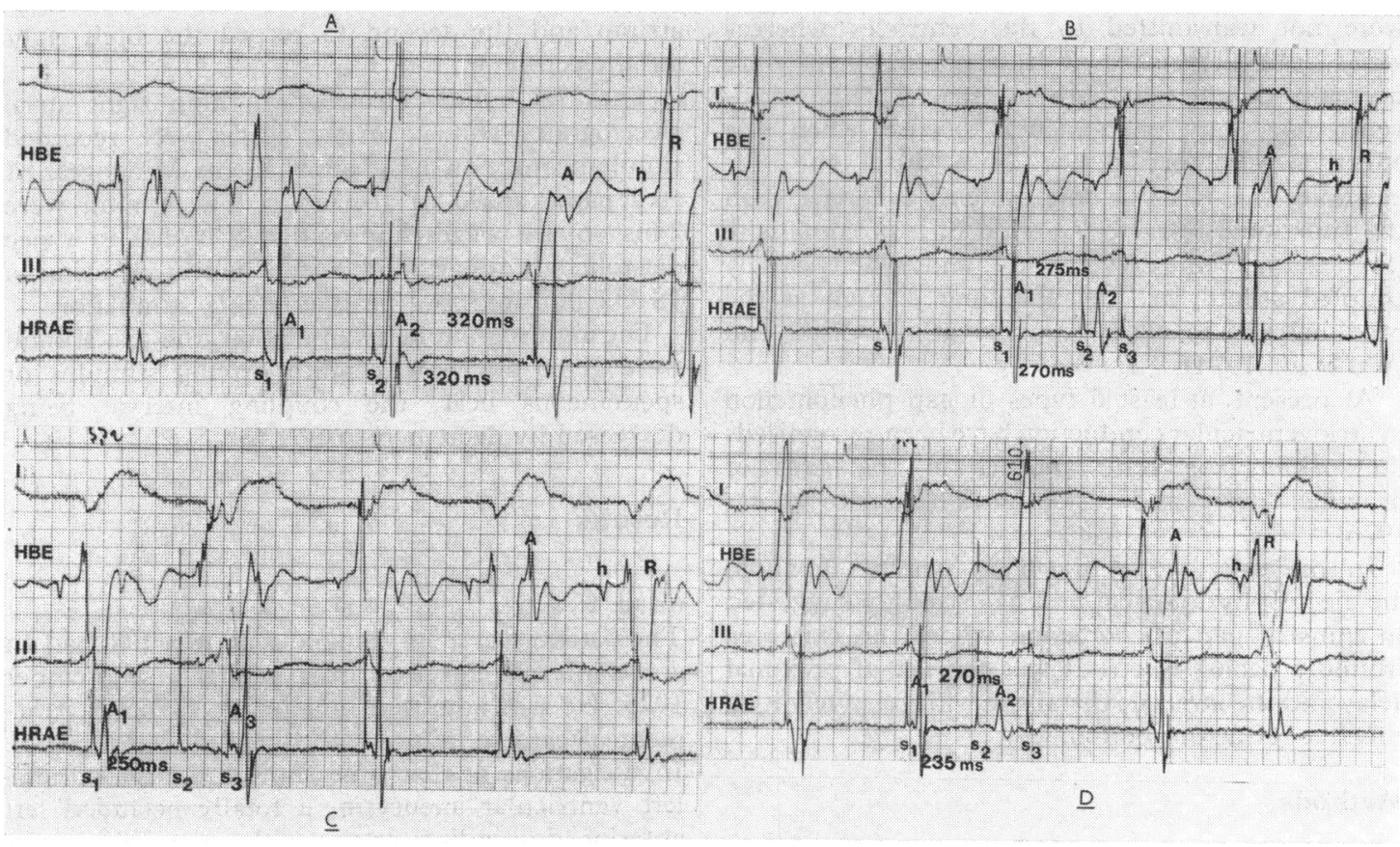

Fig. 1 Records of leads $I$ and III of the standard electrocardiogram, and of the His bundle (HBE) and high right atrial (HRAE) electrograms. Paper speed $100 \mathrm{~mm} / \mathrm{s}$. Basic driving cycle length $400 \mathrm{~ms}$. (A) At an S1-S2 interval of $320 \mathrm{~ms}$, the interval between the atrial responses of the HRAE was also $320 \mathrm{~ms}$. (B) At an S1-S2 of $270 \mathrm{~ms}$, the corresponding A1-A2 interval was slightly longer (275 ms) indicating a small prolongation of the latency time between stimulus artefact and atrial deflection. (C) The stimulus falling at $250 \mathrm{~ms}$ evoked no atrial response, though the next stimulus (S3) was conducted. (D) Following S2 placed $235 \mathrm{~ms}$ after S1, an atrial response was again observed. (See text for further description.) 


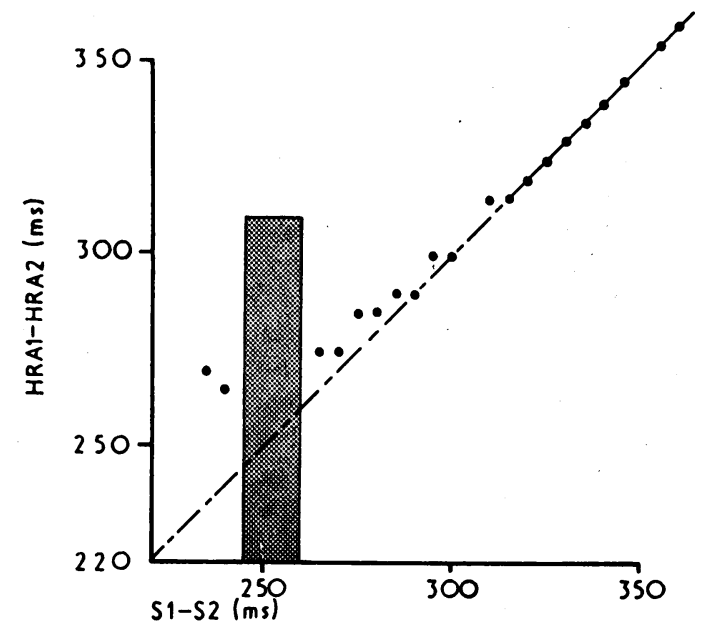

from 420 to $305 \mathrm{~ms}$. For S1-S2 times of $300 \mathrm{~ms}$ or shorter, lengthening of the S2-HRA2 delay was seen to shift the curve upward. The S2 stimuli falling 255 and $250 \mathrm{~ms}$ after $\mathrm{S} 1$ did not evoke atrial responses though the pacing stimulus (S3) falling immediately after $\mathbf{S} 2$ induced an atrial depolarisa-

Fig. 2 Case 1. S1-S2 intervals plotted against HRA1-HRA2 intervals. Basic cycle length $400 \mathrm{~ms}$ $(150 / \mathrm{min})$. The line of identity was followed for S1-S2 intervals of 360 to $290 \mathrm{~ms}$. A gap in atrial responses was observed at S1-S2 intervals of 260, 250, and $245 \mathrm{~ms}$. Atrial excitation resumed at S1-S2 intervals of 240 and $235 \mathrm{~ms}$. Beyond this point, no further atrial response could be evoked. Note that the last two HRA1-HRA2 intervals were approximately as long as those seen before the gap in atrial responses.

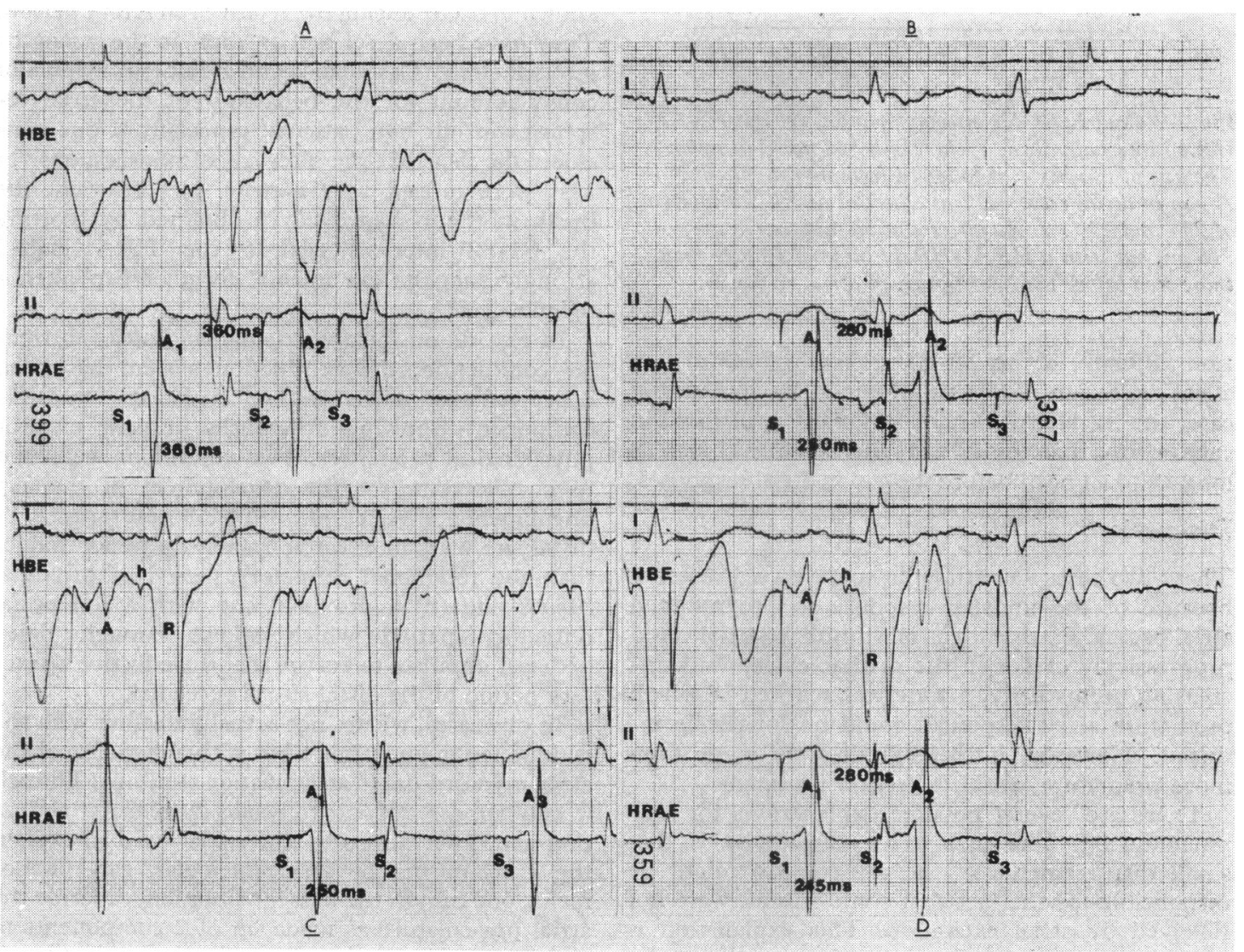

Fig. 3 Case 2. Leads I and II are shown with the HBE and HRAE. Paper speed is $100 \mathrm{~mm} / \mathrm{s}$. The basic driving length is $545 \mathrm{~ms}$. At an S1-S2 interval of $360 \mathrm{~ms}(A)$, the distance between the atrial responses of the $H R A E$ was also $360 \mathrm{~ms}$. At an S1-S2 of $260 \mathrm{~ms}(B)$, the corresponding A1-A2 interval was 280 ms indicating a prolongation of latency time between stimulus artefact and atrial deflection. The atrial stimulus falling 250 ms after $S 2$ (C) failed io evoke any atrial response but the next extrastimulus succeeded in stimulating the atrial chambers (D). 


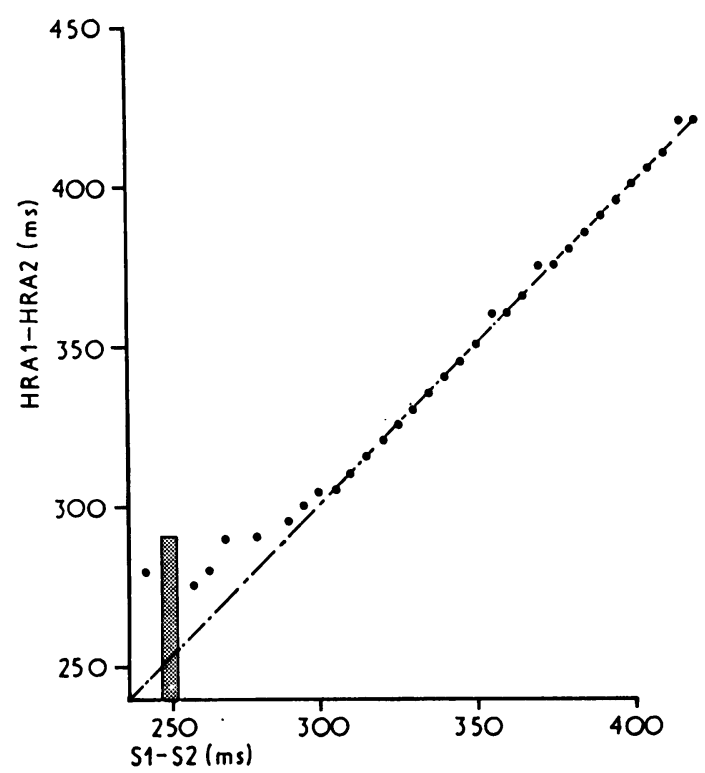

Fig. 4 Case 2. S1-S2 intervals plotted against HRA1HRA2 intervals. Basic cycle length $545 \mathrm{~ms}$ (110/min). The line of identity was followed from 420 to $305 \mathrm{~ms}$. $A$ gap in atrial responses was observed at S1-S2 intervals of 255 and $250 \mathrm{~ms}$. Atrial excitation reappeared at 245 ms, but with a HRA1-HRA2 interval greater than those seen before the interruption in atrial responses.

tion. Finally, at an S1-S2 interval of $245 \mathrm{~ms}$, an atrial deflection was again obtained; as in the first case, the S2-HRA2 delay was greatly prolonged in such a way that the HRA1-HRA2 interval was as long as it had been before the gap in atrial responses.

\section{Discussion}

These two cases were thought to deserve discussion because of the unusual response to programmed atrial pacing. Premature atrial stimuli were delivered progressively earlier in the cardiac cycle. With increasing prematurity, a zone was reached in which premature atrial responses could no longer be induced. However, if the stimuli were made even more premature, atrial excitation resumed.

There are several possible explanations for this phenomenon. The first to be considered is that small displacements of the catheter tip might be responsible for the fact that some stimuli were not followed by atrial excitation. This explanation is unlikely: in these two cases the phenomenon under discussion occurred only at a critical moment of the cardiac cycle close to the end of the atrial effective refractory period; moreover, the driving stimulus, which directly followed the impulse which was not conducted, did induce a normal atrial response. Another possible explanation is a phase of supernormal excitability. Though supernormal excitability and conduction has been documented in animal and human experiments, intracellular stimulation methods indicate that not all cardiac cells can exhibit this property (Spear and Moore, 1976). Supernormal excitability has been observed in the His-Purkinje system (Spear and Moore, 1974) and in specialised atrial fibres (Childers, 1974) and in specialised atrial fibres (Childers et al., 1968). It has not been documented in the AV node (Merideth et al., 1968), the bundle of His (Spear and Moore, 1974), or in atrial or ventricular muscle (Childers et al., 1968; Spear and Moore, 1974). In our 2 cases, one cannot rule out the possibility of direct stimulation of specialised atrial fibres by the spacing electrode. In addition to the classical supernormal phase, minimal changes of excitability are known to occur during the relative refractory period. They have been described as 'dips' in the strengthinterval curve (Castellanos and Lemberg, 1969) and could account for the behaviour of the atrial responses in our two patients, especially if one considers the fact that the intervals of non-responsiveness were indeed very narrow. Nevertheless, the break in the J-shaped curve obtained by plotting the S1-S2 intervals against the HRA1-HRA2 strongly suggests an unusual form of gap phenomenon.

In reports on intraventricular and atrioventricular conduction, the conduction system has been conceived as a linear structure consisting of multiple components showing differences in their electrophysiological properties. Two conditions were said to be necessary for the development of the gap phenomenon (Wu et al., 1974). These are (a) a distal site with an effective refractory period longer than the functional refractory period of proximal tissues and (b) a proximal site with a functional refractory period which allows enough delay between impulses to ensure that the effective refractory period of the distal site is exceeded.

In our cases, we are not actually dealing with the conduction of an impulse but with a response of the atrial myocardium to an activation stimulus. Though it may appear difficult to relate these atrial activation gap observations to the model used to explain the mechanisms of atrioventricular gap phenomena, one can, in the present situation, describe the atrial musculature as made up of 2 components in series: the exit area around the catheter tip (proximal site) and the rest of the atrial myocardium (distal site).

The exit area from a pacing electrode is known to share some properties with conducting tissues. Exit 
block around a pacing electrode has been recognised (Preston et al., 1966), and may be manifested as a Wenckebach phenomenon (Peter et al., 1976), or as a gradual prolongation of exit time from a pacemaker potential (Pick, 1973). Increase in latency time between the stimulus artefact and the high right atrial deflection has also been reported, during programmed atrial stimulation, when the coupling intervals are sufficiently short (Wu et al., 1974).

In our cases, as the S1-S2 interval was decreased the HRA1-HRA2 intervals followed the line of identity along its upper portion. With further decrease in S1-S2 interval, the HRA1-HRA2 interval continued to decrease but to a lesser extent, indicating an increase of latency time (S2-HRA2). This feature can be interpreted as showing increasing first degree exit block around the pacing electrode. To account for the reappearance of atrial responses after the interruption of the J-shaped curve, one can reasonably hypothesise that at a critical S1-S2 interval, the proximal delay in the exit area could be sufficiently long for some stimuli apparently falling within the atrial effective refractory period to activate the atria.

If this interpretation is correct the two reported cases would represent unusual examples of an intra-atrial gap which to the best of our knowledge, has not hitherto been described.

\section{References}

Castellanos, A., Jr., and Lemberg, L. (1969). Electrophysiology of Pacing and Cardioversion. Butterworths, London.
Childers, R. W., Merideth, J., and Moe, G. K. (1968). Supernormality in Bachmann's bundle. An in vitro and in vivo study in the dog. Circulation Research, 22, 363.

Damato, A. N., Akhtar, M., Ruskin, J., Caracta, A., and Lau, S. H. (1976). Gap phenomenon: antegrade and retrograde. In The Conduction System of the Heart, pp. 504-528. Ed. by H. J. J. Wellens, K. I. Lie, and M. J. Janse. H. E. Stenfert Kroese B.V., Leiden.

Merideth, J., Mendez, C., Mueller, W. J., and Moe, G. K. (1968). Electrical excitability of atrioventricular nodal cells. Circulation Research, 23, 69.

Moe, G. K., Mendez, C., and Han, J. (1965). Aberrant A-V impulse propagation in the dog heart. A study of functional bundle branch block. Circulation Research, 16, 261.

Peter, Th., Harper, R., Hunt, D., and Sloman, G. (1976). Wenckebach phenomenon in the exit area from a transvenous pacing electrode. British Heart fournal, 38, 201.

Pick, A. (1973). Mechanisms of cardiac arrhythmias: from hypothesis to physiologic fact. American Heart fournal, 86, 249.

Preston, T. A., Judge, R. D., Bowers, D. L., and Morris, J. D. (1966). Measurement of pacemaker performance. American Heart fournal, 71, 92.

Scherlag, B. J., Lau, S. H., Helfant, R. H., Berkowitz, W. D., Stein, E., and Damato, A. N. (1969). Catheter technique for recording His bundle activity in man. Circulation, 39, 13.

Spear, J. F., and Moore, E. N. (1974). Supernormal excitability and conduction in the His-Purkinje system of the dog. Circulation Research, 35, 782.

Spear, J. F., and Moore, E. N. (1976). Supernormal excitability and conduction. In The Conduction System of the Heart, pp. 111-125. Ed. by H. J. J. Wellens, K. I. Lie, and M. J. Janse. H. E. Stenfert Kroese B.V., Leiden.

Wu, D., Denes, P., Dhinghra, R., and Rosen, K. M. (1974). Nature of the gap phenomenon in man. Circulation Research, 34, 682.

Requests for reprints to Dr. H. E. Kulbertus, Division of Cardiology, University Hospital, 66 Boulevard de la Constitution, B-4020-Liège, Belgium. 